\title{
Cerebellar dysregulation and heterogeneity of mood disorders
}

This article was published in the following Dove Press journal:

Neuropsychiatric Disease and Treatment

23 July 2014

Number of times this article has been viewed

\section{Edward $\mathrm{H}$ Tobe}

Department of Psychiatry, Cooper Medical School of Rowan University, Camden, NJ, USA
Correspondence: Edward H Tobe I00 I Lincoln Drive West, Suite B, Marlton, NJ 08053-I534, USA

Tel + I 8569834940

Fax + I 8569833408

Email edward.tobe@comcast.net
Abstract: This paper discusses diverse studies to consider the hypothesis that cerebellar pathology supports the heterogeneous metabolic pathologies of mood disorders. The evidence presented includes studies selected from the following areas of scientific research: magnetic resonance imaging, histology, clinical syndromes, comparative anatomy, neuronal connections, and mitochondrial dysfunction. The gamut of different scientific study methods confirms the validity of the involvement of the cerebellum in mood disorders.

Keywords: psychiatry, neurology, depression, cerebellum, neuropathology, heterogeneity

\section{Introduction}

Early studies of mental illness failed to correlate psychologic findings with anatomic changes in the central nervous system. ${ }^{1}$ The failure to establish reproducible biologic markers with specificity and selectivity may reflect the heterogeneity of mood disorders. Scholarly research in the 19th century debated brain localization and the relationship between dementia, depression, and paralysis. ${ }^{2}$ Temporal and frontal lobe pathology has been the major focus of biologic research.

The biogenic amine theory of depression began with the observation of mood elevation in some patients with tuberculosis treated with iproniazid. ${ }^{3}$ The catecholamine hypothesis of mood disorders assumes that the effect of a drug on symptoms is more than of heuristic value (post hoc ergo propter hoc). ${ }^{4}$ In a meta-analysis of six studies (including a total of 718 patients) with severity of illness rated by the Hamilton Depression Rating Scale ( $\leq 18$, mild to moderate depression; 19-22, severe depression; $\geq 23$ very severe depression), only scores $\geq 23$ could differentiate between placebo and minimal response to medication. ${ }^{5}$

Science will advance the diversity of research. Historically, medicine considered the cerebellum as a modulator of motor function. Different study approaches have enhanced our appreciation of the complex bilateral relationship between the cerebrum and the cerebellum. The purpose of this paper is to describe diverse scientific approaches to evaluate the hypothesis that cerebellar pathology may affect the heterogeneous metabolic pathologies of mood disorders. To accomplish this aim, data are presented from different methods of study. Each method supplies evidence that when accumulated supports the significance of the cerebellum in mood disorders.

\section{Evidence Clinical syndromes}

More than half of childhood brain tumors originate in the posterior fossa, which contains the cerebellum and brainstem. A postoperative syndrome develops in some 
children, and is characterized by dysphagia, cranial nerve palsies, diminished motor movement, and speech disturbance, including mutism. This posterior fossa syndrome may last months before resolving.

Schmahmann studied five children who developed posterior fossa syndrome after the resection of a midline cerebellar tumor. ${ }^{6}$ These patients became mute on postoperative day 1 to 4 , and recovery required several months. During the recovery period, the patients exhibited dysarthria, buccal and lingual apraxia, and behavioral changes, including regressive personality changes, apathy, withdrawal, limited spontaneous movement, and emotional lability (periods of irritability, crying, agitation, giggling, and distractibility). ${ }^{6}$

The presentation of these five pediatric patients demonstrated the cerebellar cognitive affective syndrome, characterized by decreased intellectual functions including: disturbed executive function, such as planning, set-shifting from one task to another, abstract reasoning, working memory, and verbal fluency; impaired spatial cognition, such as visuospatial organization and memory; personality change, including flat affect and disinhibited behavior; and linguistic dysfunction, including changes in speech patterns, broken grammar, and difficulty in using words about objects. ${ }^{7}$ Cerebellar cognitive affective syndrome suggests:

Disruption of the cerebellar modulation of neural circuits that link prefrontal, posterior parietal, superior temporal and limbic cortices with the cerebellum. ${ }^{7}$

Reversed cerebellar diaschisis documented by areas of hypoperfusion in cerebral association areas was seen on PET/SPECT of the three patients. ${ }^{7}$

\section{Comparative anatomy}

Recognition of the cerebellum as contributing to mental skills was encouraged by anatomic dissection, demonstrating in $1986^{8}$ a large connection of fibers between the cerebellum and cognitive areas of the cerebral cortex, ${ }^{9}$ which questioned the purpose of a large fiber connection. ${ }^{10} \mathrm{Com}-$ parison of the cerebellar-cerebral anatomy of humans and other species found that humans have a large tract of cerebellar-cerebral connection; yet other species, such as monkeys, who possess extraordinary motor skills, do not share this similarity. Anthropoid apes and humans share a common phylogeny of significant enlargement of the lateral cerebellum. ${ }^{11}$ Excluding cats, the cerebellum in higher primates is connected to the cerebral cortex in a different way from that in nonprimates. Nerve fibers connect to an enlarged association area in the frontal lobe. The dentate nucleus is larger in apes and humans than in other species, especially the phylogenetic newer ventrolateral dentate. The evolution of the dentate is associated with a parallel evolution of the expansion of the prefrontal cortex in apes and humans, but not in other species.

The cerebellum connects with multiple subcortical structures, including the vestibular nuclei and basal ganglia. Projections from the cerebellum to the cerebral cortex first synapse in the dentate, then synapse in the contralateral thalamus and project to the cerebral cortex. Cerebral cortex projections to the cerebellum first synapse in the ipsilateral anterior pontine nuclei and then cross to the contralateral cerebellar cortex. The basal ganglia project to the subthalamic nucleus and then to the pons. ${ }^{12}$ The evidence increasingly argues that the cerebellum has very distinct intrinsic connective networks that relate to specific areas with specific functions. $^{12}$

\section{Magnetic resonance imaging}

Magnetic resonance imaging has offered anatomic evidence of cerebellar pathology and mood disorders. Among the studies demonstrating this correlation, a magnetic resonance imaging (MRI) study compared hospitalized manic patients (initial manic episode, 16 patients; prior manic episodes, 14 patients) and control subjects (15 patients). ${ }^{13}$ The right and left cerebellar vermal areas 1-3 were evaluated. Cerebellar vermal area 3 (vermal lobules VIII-X) was smaller in patients who had bipolar disorder and in those who experienced multiple episodes of depression. This study was limited in the number of patients included, and the results may have been confounded by other factors, such as psychotropic medication or recurrent depression. Elimination of substance abusers in the 3-month period before the study did not change the results. This study suggests further scrutiny into whether cerebellar vermal atrophy may be a late neurodegenerative event in patients who have multiple affective episodes. ${ }^{14}$

Functional MRI has been used to study neural activity in the cerebellum. Thirty healthy subjects aged 18-30 years were studied to explore evidence of a specific "primary emotion" response to stimulation. Images were provided that used the International Affective Picture System to characterize what the author refers to as "primary emotions", ie, happiness, anger, disgust, fear, and sadness. All five emotions evoked spatially distinct patterns of activity in the posterior lobe of the cerebellum; however, there were areas of overlap between cerebellar activation, suggesting, according to the authors, "some shared neural networks". An alternative hypothesis to explain the shared areas is that functional MRI demonstrates the limitations of the International Affective Picture System. ${ }^{13}$ 


\section{Case study with histology}

A previously healthy middle-aged man sustained a closed head injury. He developed progressive depression, volatile behavior, dementia, dysphagia, and choppy speech. ${ }^{15} \mathrm{He}$ intimidated women, walked the public streets with feces on his clothing, and resisted police arrest after publically almost beating the family dog to death. The court determined that he was incompetent in self-care. While involuntarily hospitalized, he died of hypoxia from a generalized tonic-clonic seizure. Microscopic evaluation showed chronic incomplete necrosis of the unilateral temporal cortex and hippocampus. The neuropil was preserved, but there was a prominent glial reaction. The cortical architecture was disorganized, with primarily smaller neurons and few pyramidal cells present. A large, disorganized collection of large neurons and granular cells was noted in the cerebellar vermis nodule. Excessive numbers of Bergmann astrocytes, which are radial glial cells existing only in the adult cerebellum, were present. ${ }^{15}$

In contrast, patients treated for cancer with radiation therapy may have inadvertent temporal lobe radiation exposure and develop depression, irritability, and dementia, including impairment of memory, concentration, language, and motor and executive ability. ${ }^{15}$ These patients do not develop aggressive psychotic behavior, choppy speech, or dysphagia. Partial necrosis of the temporal lobe does not explain the presentation; rather, the combination of temporal and cerebellar pathology and/or the connection between the cerebellum and temporal lobe affect the outcome of the illness.

\section{Anatomic evidence}

Middleton et al studied the neuronal connections between the dorsolateral prefrontal cortex and the dentate nucleus, thalamus, and basal ganglia. ${ }^{12}$ They injected the McIntyre strain of herpes simplex virus type 1 into dorsolateral prefrontal cortex area 46 of three Cebus paella (cebus) monkeys. This cortical area was selected because it is a nonmotor region of the frontal lobe. Once injected, the virus was transported within the neuron in a retrograde direction. The virus was traced to three thalamic nuclei known to innervate the dorsolateral prefrontal cortex, ie, the ventralis anterior pars parvocellularis, the medialis dorsalis, and the ventralis lateralis pars caudalis. The virus was transported predominantly to the contralateral (versus ipsilateral) dentate nucleus, to a location within the dentate nucleus not associated with motor function. The ipsilateral internal portion of the globus pallidus received the virus in a region of the globus pallidus not associated with motor movement. The authors successfully showed connections between particular areas of the cortex, cerebellum, basal ganglia, and thalamus that are not associated with motor function. ${ }^{12}$

\section{Mitochondrial dysregulation}

The cerebellum holds over half of the total neurons comprising the brain. These neurons require a stable energy source derived from mitochondria. According to the energy need in a section of the neuron, mitochondria travel along cytoskeletal structures. Perturbations in the dynamic metabolism of mitochondria compromise mitochondrial membrane permeability and threaten cellular neuroplasticity. Among the important enzyme regulators of the intracellular microenvironment are monoamine oxidase (MAO) and superoxide dismutase. These enzymes are significant in maintaining a delicate balance of reactive oxidative species. Oxidative stress may cause mitochondrial fragmentation.

MAO is an enzyme attached to the outer membrane of the mitochondrion and oxidizes biogenic amines. In purified bovine brain mitochondria, the function of MAO may vary with the microenvironment ( $\mathrm{pH}$, ion concentration, and heat) and this may affect substrate metabolism (lipids, proteins, and carbohydrates). ${ }^{16} \mathrm{MAO}$ is present throughout brain tissue. There are two types of MAO, ie, type A (MAO-A) and type $\mathrm{B}$ (MAO-B). Although MAO-A is the only MAO located in the gut, both MAO-A and MAO-B exist in the central nervous system. As people age, the concentration of MAO-A does not change but MAO-B increases. ${ }^{17}$

A study of MAO-B performed with positron emission tomography in healthy male subjects (aged 27, 43, and 86 years), found that increased age was associated with increased MAO-B in most areas of the brain, including the thalamus, basal ganglia, ventral striatum, cerebellum, and pons, but not the cingulate gyrus. ${ }^{17}$ With advancing age and increasing amounts of MAO-B attached to the outer mitochondrial membrane, enhanced oxidation of monoamines may cause elevated levels of reactive oxygen species that may threaten cellular viability. The United States Food and Drug Administration approved selegiline, a selective MAO-B inhibitor (when taken in low oral doses) for the treatment of Parkinson's disease, a synucleinopathy characterized by pathologic aggregation of $\alpha$-synuclein to form insoluble fibrils. Excessive reactive oxygen species cause $\alpha$-synuclein to increase in the synaptic intraneuronal region and lose solubility.

There is a delicate intracellular balance between free radicals that serve as messengers or cause oxidative damage to mitochondrial and nuclear DNA. Intracellular antioxidants are important in defense of the cell against excessive reactive oxygen species. Superoxide dismutase may protect intracellular metabolism from oxidative stress. There are three forms of superoxide dismutase in humans. Glutathione peroxidase is a superoxide dismutase that reduces lipid hydroperoxides and scavenges peroxides. 
In an experiment evaluating the effect of selegiline on antioxidant function in adult rats, the level of peroxides in cerebellar tissue was greater in older rats (aged 12 and 18 months) than in younger rats (aged 6 months) treated with or without selegiline. ${ }^{18}$ This suggests that increased age may be associated with a decrease in the antioxidant capacity of the cell. Rats that did not receive selegiline had a decreased concentration of antiperoxidase enzymes, and rats that received selegiline had higher levels of antiperoxidase and less lipid peroxidase.

The combination of increased MAO-B concentration and decreased antioxidant enzymes with advancing age could explain the neurologic, cognitive, affective, and behavioral dysfunction that may occur with increased age, as observed in synucleinopathies and tauopathies. Alterations in the capacity to modulate mood and motor function plus impairment of cognition suggest that dysregulation of the cerebellum is a significant factor, along with the cerebrum in the presentation of disease.

\section{Conclusion}

Mood disorders may be very disabling. Current disease classification does not provide the practitioner with an accurate biologic diagnosis. Approaches to the treatment of mood disorders have focused on the telencephalon. However, cerebellar dysfunction may have primary or secondary effects on mood disorders.

The human brain has evolved with anatomic evidence of a complementary relationship between the cerebellum and the prefrontal cortex. Metabolically active bidirectional connections have been defined between the cerebellum, thalamus, basal ganglia, and cortex. Cerebellar disease may present with behavioral, affective, and cognitive abnormalities. The age-related increase in MAO-B levels and decreased intracellular antioxidants may cause increased reactive oxygen species and associated cellular dysregulation. The evidence that the cerebellum may contribute to the heterogeneity of mood disorders includes data from comparative anatomic, clinical, histologic, metabolic, and imaging studies and observations about cerebellar diseases that have associated psychiatric findings. Further study is justified to clarify the contribution of the cerebellum to the regulation of cognition, affect, and behavior.

\section{Disclosure}

The author reports no conflict of interest in this work.

\section{References}

1. Maj M. Mental disorders as "brain diseases" and Jaspers' legacy. World Psychiatry. 2013;12(1):1-3.

2. Berrios GE. "Depressive pseudodementia" or "melancholic dementia": a 19th century view. J Neurol Neurosurg Psychiatry. 1985;48(5):393-400.

3. Pletscher A. The discovery of antidepressants: a winding path. Experientia. 1991;47(1):4-8.

4. Schildkraut JJ. The catecholamine hypothesis of affective disorders: a review of supporting evidence. Am J Psychiatry. 1965;122(5):509-522.

5. Fournier JC, DeRubeis RJ, Hollon SD, et al. Antidepressant drug effects and depression severity: a patient-level meta-analysis. JAMA. 2010;303(1):47-53.

6. Schmahmann JD. Disorders of the cerebellum: ataxia, dysmetria of thought, and the cerebellar cognitive affective syndrome. J Neuropsychiatry Clin Neurosci. 2004;16(13):367-378.

7. Schmahmann JD, Sherman JC. The cerebellar cognitive affective syndrome. Brain. 1998;121 Pt 4:561-579.

8. Leiner HC, Leiner AL, Dow RS. Does the cerebellum contribute to mental skills? Behav Neurosci. 1986;100(4):443-454.

9. Leiner HC, Leiner AL, Dow RS. Reappraising the cerebellum: what does the hindbrain contribute to the forebrain? Behav Neurosci. 1989;103(5): 998-1008.

10. Leiner HC, Leiner AL, Dow RS. Cognitive and language functions of the human cerebellum. Trends Neurosci. 1993;16:444-447.

11. D'Angelo E, Casali S. Seeking a unified framework for cerebellar function and dysfunction: from circuit operations to cognition. Front Neural Circuits. 2013;6:116.

12. Middleton FA, Strick PL. Anatomical evidence for cerebellar and basal ganglia involvement in higher cognitive function. Science. 1994; 266(5184):458-461.

13. Baumann O, Mattingley JB. Functional topography of primary emotion processing in the human cerebellum. Neuroimage. 2012;61(4): 805-811.

14. DelBello MP, Strakowski SM, Zimmerman ME, Hawkins JM, Sax KW. MRI analysis of the cerebellum in bipolar disorder: a pilot study. Neuropsychopharmacology. 1999;21(1):63-68.

15. Tobe EH. Behavioral effects of incomplete temporal lobe necrosis and cerebellar damage. Biol Psychiatry. 2012;71:e41-e42.

16. Gabay S, Achee FM, Mentes G. Some parameters affecting the activity of monoamine oxidase in purified bovine brain mitochondria. J Neurochem. 1976;27(2):415-424.

17. Fowler JS, Volkow ND, Wang GJ, et al. Age-related increases in brain monoamine oxidase B in living healthy human subjects. Neurobiol Aging. 1997;18(4):431-435.

18. Subramanian MV, James TJ. Age-related protective effect of deprenyl on changes in the levels of diagnostic marker enzymes and antioxidant defense enzymes activities in cerebellar tissue in Wistar rats. Cell Stress Chaperones. 2010;15(5):743-751.
Neuropsychiatric Disease and Treatment

\section{Publish your work in this journal}

Neuropsychiatric Disease and Treatment is an international, peerreviewed journal of clinical therapeutics and pharmacology focusing on concise rapid reporting of clinical or pre-clinical studies on a range of neuropsychiatric and neurological disorders. This journal is indexed on PubMed Central, the 'PsycINFO' database and CAS,

\section{Dovepress}

and is the official journal of The International Neuropsychiatric Association (INA). The manuscript management system is completely online and includes a very quick and fair peer-review system, which is all easy to use. Visit http://www.dovepress.com/testimonials.php to read real quotes from published authors. 